\title{
A CLASSIFICATION OF INTERNET PORNOGRAPHIC IMAGES
}

\author{
Chetneti Srisa-an \\ Rangsit University \\ 52/347 Muang Ake, Phaholyothin Rd., Pathumthani 12000, Thailand \\ chetneti@rsu.ac.th
}

\begin{abstract}
Internet pornography (Internet Porn) is addictive to teenagers and kids around the world. The normal practice is to block those websites or filter out pornography from kids. Many research papers have been published how to detect a human pornographic image on web pages. A new technique was proposed here to classify pornography images using range of $\mathrm{YCbCr}$ (colour feature) and three new measurements: \%Face_Area, \%AHB and $\mathrm{R}_{\max }$. A computer algorithm such as $\mathrm{C} 4.5$ was applied to construct a decision tree. The main contribution of this paper is simplicity that retains high accuracy within an acceptable processing time. The accuracy of experimental results was $85.2 \%$ with an average processing time of 0.21314 seconds per image.
\end{abstract}

Keywords: Pornographic images, Data Classification, Skin Detection, Internet Porn

\section{INTRODUCTION}

Pornographic images on the Internet has become a serious problem for parents around the world. To address this issue, the normal practice is to block websites or filter out nude images from web pages. This paper presents a strategy to detect pornographic images. Firstly, if nudity means the state of being without or having little coverage with clothing, then range of $\mathrm{YCbCr}$ is a good way to detect human skin in images.

Detecting a face using color information is the second step. According to Chai and $\mathrm{Ngan}^{1}$, face location can be identified by performing region segmentation with the use of a skin color map. The presence of $\mathrm{Cr}$ values within, 136 and 156, and $\mathrm{Cb}$ values within 110 and 123 indicates a human face region. After performing face detection, if the largest face area is greater than the specified threshold, the image is classified as a non-nude image $^{2}$. In this paper, we classify this type of picture as a Selfie/close-up face type. The first measurement called \%Face_Area is defined in step 2 
(shown in section 3.2). If it is not a close-up image, then we can calculate a $\%$ AHB (Area of Human Body) value by expanding the scope of $\mathrm{YCbCr}$ to a full range as shown in section 3.3.

This program was developed by utilizing MATLAB software, Version 7.1 on 15,000 sample images with an average size of 405,318 pixels or 86 $\mathrm{Kb}$. The experimental results showed that accuracy was $85.2 \%$. Average processing time was $213.14 \mathrm{msec}$. per image

This paper is organized as follows. Section 2 presents the literature review. Our new methodology is presented in section 3. Subsequently, in section 4, the experimental results are presented. Finally, we conclude our work with conclusions and future work in section 5.

\section{LITERATURE REVIEW}

Several methods have been recently developed to detect and classify nude images.

Chai and Ngan ${ }^{1}$ pointed out that chrominance values in the facial region can be used as a classifier. Therefore, this individual color feature can simply be defined by the presence of $\mathrm{Cr}$ values within, for example, 136 and 156, and $\mathrm{Cb}$ values within 110 and 123. They also indicated that color is a powerful descriptor that has practical use in the extraction of face location. The color information is typically used for region rather than edge segmentation.

M.Z. Talukder et al. ${ }^{2}$ presented that a skin surface reflects light in a different way as compared to other surfaces. A skin region can be classified by comparing large image content of a skin database and non-skin database. The RGB color space is widely used and most effective for detecting the skin region from an image.

Leea et al. ${ }^{3}$ stated that warm lighting can help skin tone to be more attractive, and a feasible solution for the problem is to adapt the adopted skin chroma distribution to the lighting of the input image.

Shin et al. ${ }^{4}$ proposed that $\mathrm{YCbCr}$ is the best color space for skin detection. The RGB color space gives the best performance in 2D. By applying a YCbCr color space, a human skin segment can be distinguished from non-skin segment.

In 2014, Blekasc et al. ${ }^{5}$ proposed that skin color information using Regions of Interest (ROIs) was useful information for detection of pornographic images.

Phung et al. ${ }^{6}$ proposed that a skin color model based on $\mathrm{Cb}$ and $\mathrm{Cr}$ values can provide good coverage of all human races. They presented a skin 
color-based face detection algorithm that employed a human skin color model, which took into account luminance $\mathrm{Y}$ in classifying skin and non-skin pixels.

\section{METHODOLOGY}

Since most web page images are 2D color images, this paper focused only on 2D color nude images. The RGB model was the first model for a $2 \mathrm{D}$ graphic image. A picture in RGB can be described as a matrix with a size of $\mathrm{m} \times \mathrm{n}$ pixels and 3 color channels: red, green and blue.

Color images typically elicit a sexual response from humans. In contrast, black and (gray) nude images might not. As such, gray images were left as out of scope in this paper.

The YCbCr color model was originally used for digital video. In $\mathrm{YCbCr}$, Y refers to the luminance component. The $\mathrm{Cr}$ and $\mathrm{Cb}$ components refer to red and blue chrominance. Skin tone is formed by the interaction between skin color and light ${ }^{3}$. Since most photographers usually use special lighting and color alteration in order to make an image look more attractive ${ }^{3}$, $\mathrm{YCbCr}$ is the best feature for detecting human $\operatorname{skin}^{7}$.

\subsection{Step 1: Detect Human Skin Area Using YCbCr Range}

$\mathrm{YCbCr}$ was examined for its effectiveness in detecting human skin color. All images are loaded into MATLAB and then were converted from $\mathrm{RGB}$ to $\mathrm{YCbCr}$. Y values represent luminance while $\mathrm{CbCr}$ values represent chrominance.

The formula to convert from RGB to $\mathrm{YCbCr}$ is as follows.

$$
\begin{aligned}
& \mathrm{Y}=0.299 \mathrm{R}+0.587 \mathrm{G}+0.114 \mathrm{~B} \\
& \mathrm{R}-\mathrm{Y}=-1.72(\mathrm{G}-\mathrm{Y})-0.678(\mathrm{~B}-\mathrm{Y}) \\
& \mathrm{B}-\mathrm{Y}=-2.53(\mathrm{G}-\mathrm{Y})-1.47(\mathrm{R}-\mathrm{Y}) \\
& \mathrm{G}-\mathrm{Y}=-0.581(\mathrm{R}-\mathrm{Y})-0.394(\mathrm{G}-\mathrm{Y}) \\
& \mathrm{V}=(\mathrm{R}-\mathrm{Y}) / 1.14=0.877(\mathrm{R}-\mathrm{Y}) \\
& \mathrm{U}=(\mathrm{B}-\mathrm{Y}) / 2.03=0.493(\mathrm{~B}-\mathrm{Y}) \\
& \mathrm{Cb}=1.144 \mathrm{U} \\
& \mathrm{Cr}=0.813 \mathrm{~V}
\end{aligned}
$$




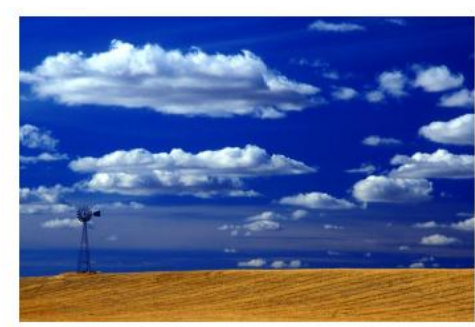

RGB

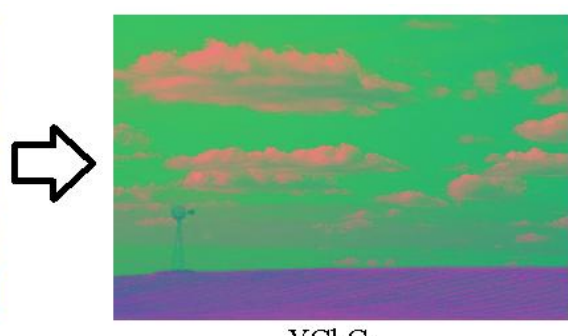

$\mathrm{YCbCr}$

Figure 1. Transformation from $\mathrm{RGB}$ to $\mathrm{YCbCr}$

Figure 1 shows a transformation from $\mathrm{RGB}$ to $\mathrm{YCbCr}$ mode. From $15,000$ samples, the range of [Y $>100],[100<\mathrm{Cb}<123$ or $128<\mathrm{Cb}<130]$ and $[130<\mathrm{Cr}<160]$ indicates a human skin. If an image was not in this range, it was not depicting human skin.

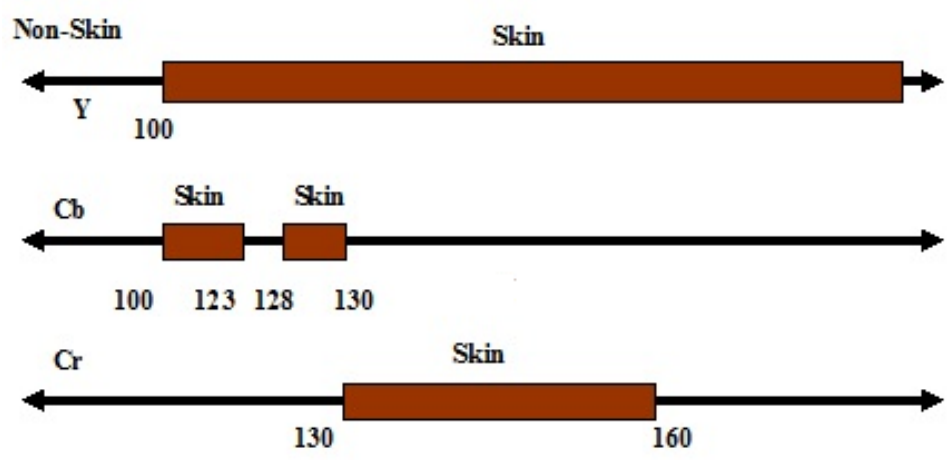

Figure 2. Human skin range

Any part of the body such as an arm, leg, chest, back or arm were applied to the same $\mathrm{YCbCr}$ range. The process was also accurate for any race including those from Asia, Europe, and Africa. Figure 2 shows a range of human skin tones.

\subsection{Step 2: Calculate a Human Face Area}

According to Chai and $\mathrm{Ngan}^{1}$, face location can be identified by performing region segmentation with the use of a skin color map. The presence of $\mathrm{Cr}$ values within 136 and 156, and $\mathrm{Cb}$ values within 110 and 123 indicate a human face region.

$$
\% \text { Face_Area }=\frac{\text { skin pixels in face segment }}{\text { total number of human skin pixels }}
$$




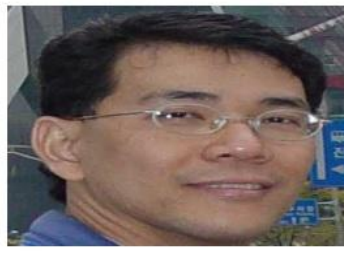

a) RGB Mode
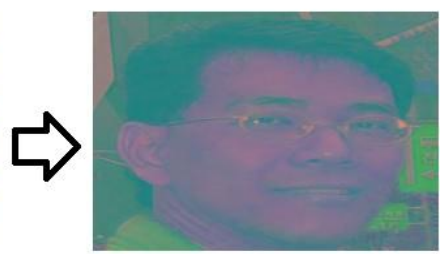

b) YCbCr Mode ( $\%$ Face_Area $=0.5009)$

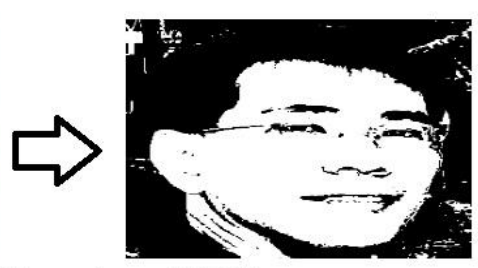

Figure 3. Close up image example

\subsubsection{Define Eccentricity}

The majority of face shapes are oval; therefore, we choose an oval shape to identify a face by considering eccentricity (Ecc).

$$
\text { Eccentricity }=\frac{\mathrm{c}}{\mathrm{a}}
$$

where $\mathrm{c}$ is the distance from the center to a focus and a is the distance from that focus to a vertex

The range of eccentricity (Ecc) is between 0 and 1 . When Ecc $=0$, it is a line (line post). When Ecc = 1, it is an oval.

Table 1 shows a sample of some raw data that is ready for constructing a decision tree.

Table 1. Sample of raw data

\begin{tabular}{llcccc}
\hline Picture\# & File name & \%Face_Area & \%AHB & Ecc & Decision \\
\hline 107 & Close_up.jpg & 0.3029 & 0.3029 & 0.5791 & No \\
278 & angelina_ph.jpg & 0.1042 & 0.4001 & 0.8794 & Yes \\
410 & CarSiam.jpg & 0.1046 & 0.3046 & 0.2919 & No \\
151 & 17988.jpg & 0.1052 & 0.4052 & 0.8985 & Yes \\
461 & pic0475.jpg & 0.1054 & 0.1054 & 0.9178 & No \\
236 & webcas12.jpg & 0.1056 & 0.4056 & 0.1913 & Yes \\
93 & akane-sakura.jpg & 0.3088 & 0.0309 & 0.9879 & No \\
30 & beauty.jpg & 0.3099 & 0.0753 & 0.3843 & No \\
371 & bauden1.jpg & 0.3014 & 0.5392 & 0.7421 & Yes \\
61 & CARN01.JPG & 0.3015 & 0.4002 & 0.6703 & No \\
433 & bg04.jpg & 0.3015 & 0.4011 & 0.501 & No \\
231 & Kiity.gif & 0.2014 & 0.4392 & 0.529 & Yes \\
444 & Japan.jpg & 0.2015 & 0.4112 & 0.601 & Yes \\
236 & Thaigirl.jpg & 0.2004 & 0.4192 & 0.901 & Yes \\
\hline
\end{tabular}




\subsection{Step 3: Calculate Percent of Human Body Area per Whole Image}

According to Chai and $\mathrm{Ngan}^{1}$, the scope of $\mathrm{Cb}$ and $\mathrm{Cr}$ in the face area is different from other parts. From step 2, we can eliminate cases for a close up or selfie image. To address a bikini case in this step, some samples of bikini cases were collected and tested. The area of Human Body (\% AHB) is the ratio of the area of human body skin (excluding a face area) compared to all the pixels of an image.

$$
\% \mathrm{AHB}=\frac{\sum_{i=1}^{\mathrm{n}}\left(\text { Area Human Body pixels in segment }_{\mathrm{i}}\right)}{\text { Total of pixels }}
$$

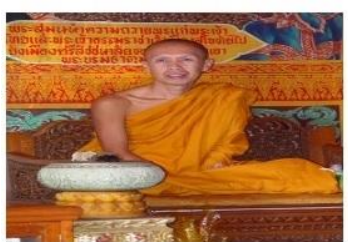

a) RGB mode

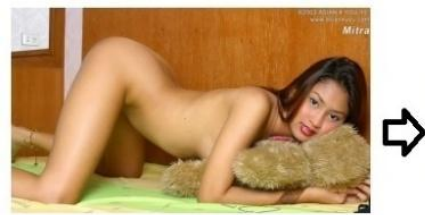

a) RGB model

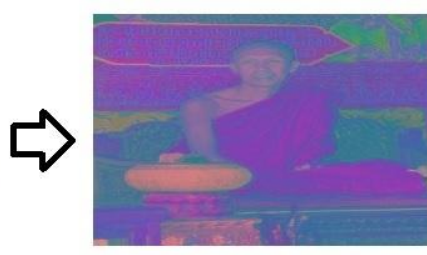

b) YCbCr mode

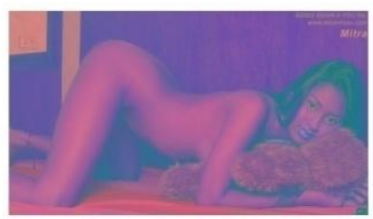

b) $\mathrm{YCbCr}(\% \mathrm{AHB}=0.438)$

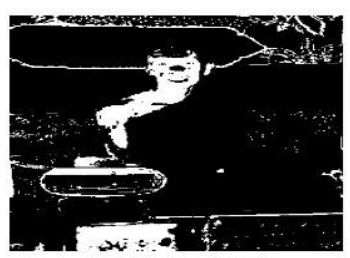

c) $\% \mathrm{AHB}=0.215$

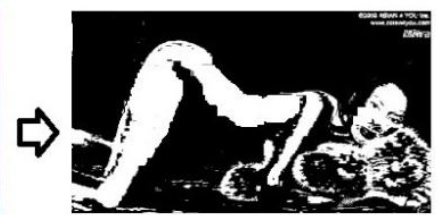

Figure 4. \%AHB sample

Figure 4 illustrates how low \%AHB leads to "not nude". The higher the $\%$ AHB, the higher the probability for a nude image.

\subsection{Constructing a Decision Tree}

Information gain is a measure that is used in information theory to quantify the information content of messages. In a $\mathrm{C} 4.5$ decision tree algorithm, information gain is used to choose the best split attribute. In this paper, information gain was used to construct the initial decision tree. By doing this, only those attributes with maximum information gain were selected for rule generation. Information gain measure was used to identify the best split attribute in a decision tree classifier. 


$$
\operatorname{Info}(\mathrm{D})=-\sum_{k=1}^{g} \frac{\operatorname{freq}(C k, D)}{|D|} * \log _{2}\left(\frac{\operatorname{freq}(C k, D)}{|D|}\right)
$$

where $|\mathrm{D}|$ is the number of transactions in database $\mathrm{D}$ and $\mathrm{g}$ is the number of classes. After the dataset $D$ is partitioned into $n$ values of attribute $A$, the expected information requirement could be defined as:

$$
\text { In fo } \mathrm{A}(\mathrm{D}) \sum \frac{\left|D_{i}\right|}{|D|} * \inf o\left(D_{i}\right)
$$

The information gained by partitioning $\mathrm{D}$ according to attribute $\mathrm{A}$ is defined as:

$$
\text { Gain }(A)=\text { info (D) }- \text { info A (D) }
$$

The best split attribute is the one that maximized the information gain in data set D. In our case, \%AHB gain was the maximum information gain.

We applied $\mathrm{C} 4.5$ to determine the cutting point of \%AHB and $\%$ Face_Area. Figure 5 shows an example of the decision tree for our model.

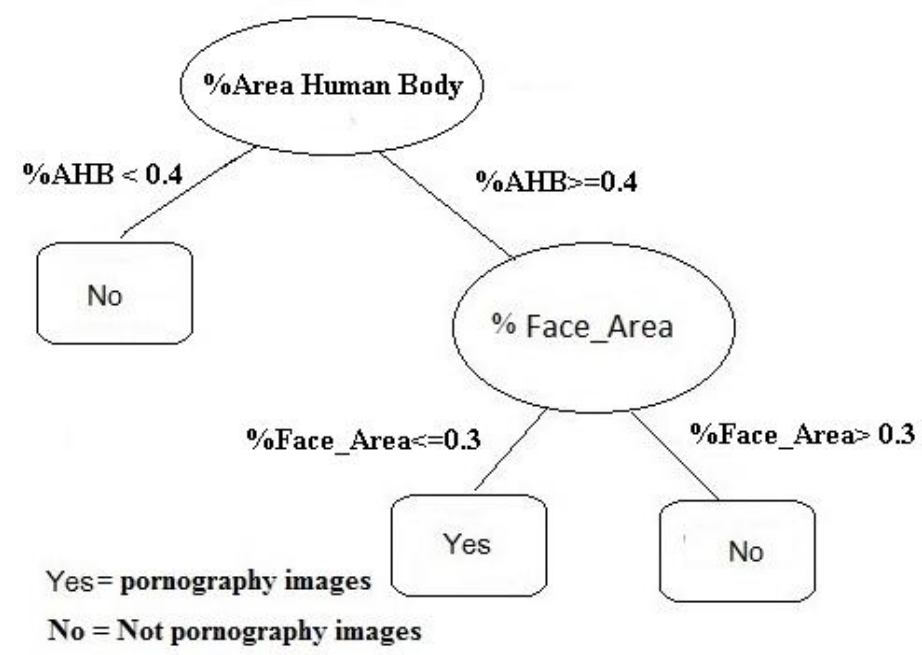

Figure 5. First model

After performing the $\mathrm{C} 4.5$ algorithm, if the human skin and the largest face area is greater than the specified threshold, the image was classified as a non-nude image. These types of images were named as "close up" images. The results show that if a human body region (excluding the face area) was greater or equal than $40 \%$ of the whole image, then this led to nude images. 


\subsection{Determine Largest \%AHB Segment $\left(R_{\max }\right)$}

We can have a pornographic image with skin region smaller than $40 \%$ of the image as shown in Figure 6. These kinds of images are considered as of the most difficult to identify in this paper. Figure 6 shows many naked passengers seated in a rollar coaster. In this case \% AHB was less than 0.4, but it was still considered a nude image. In many cases, there are many segments where many contain many $\% \mathrm{AHB} . \mathrm{R}_{\max }$ stands for the biggest $\%$ AHB segment.

$\mathrm{R}_{\max }=\max \left(\% \mathrm{AHB}_{1}, \% \mathrm{AHB}_{2} \ldots \ldots \ldots \ldots . . . \% \mathrm{AHB}_{\mathrm{n}}\right)$

If $(\% \mathrm{AHB}<0.4)$ and $\left(\mathrm{R}_{\max }>0.1\right)$ then an image is nude.

If $(\% \mathrm{AHB}<0.4)$ and $\left(\mathrm{R}_{\max }<=0.1\right)$ then an image is not nude.

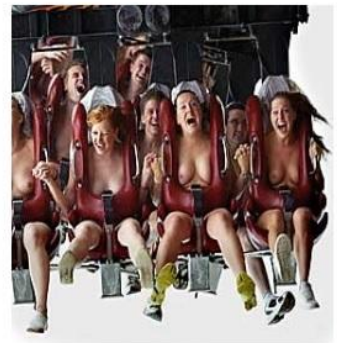

a)RGB mode
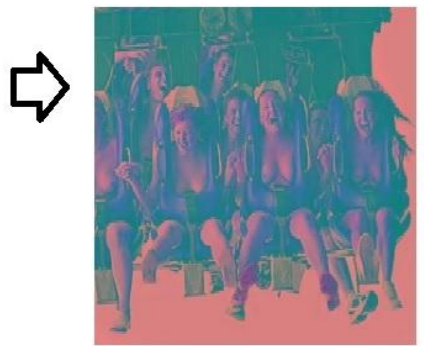

b) $\mathrm{YCbCr}$

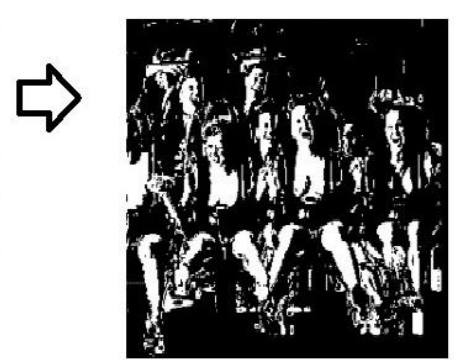

$(\mathrm{Rmax}=0.15) \quad(\% \mathrm{AHB}=0.36)$

Figure 6. Small nude picture

\section{EXPERIMENT AND RESULTS}

In our experiment, we first classified a color image collection by visual judgment. Nude/non-nude images are gathered from various web sites. The total number of nude images were 6,983 images, and the total number of non-nude images were 8,017 images. Non-nude images contained animals, people, buildings, cars, scenery, etc. Weka is a tool used for constructing a decision tree. All sample data were divided into two sets. Training data consisted of 10,000 images and the rest of the images $(5,000)$ were used for test data. The performance of the proposed algorithm can be measured by a true and false positive model. True positive (TP) and false positive (FP) are statistical measures of the performance of a binary classification test. Binary classification is the task of classifying the members of a given set of objects into two groups on the basis of whether they have some property or not.

TP is also called sensitivity, and measures the proportion of actual positives, which are correctly identified as such. Meanwhile, FP measures the proportion of actual negative that are incorrectly identified. The FP rate 
is equal to the significance level. The specificity of the test is equal to one minus the FP rate $(1-\mathrm{FP})$.

To evaluate the results of the proposed adult content detector two different metrics were used. TP (true positive) is the number of adult images identified correctly divided by the number of all test images. FP (false positive) is the number of non-adult images identified as adult images divided by the number of all control images.

Table 2. Benchmark

\begin{tabular}{ccc}
\hline Method & TP & FP \\
\hline Proposed Method & $85.2 \%$ & $4.2 \%$ \\
Arentz-Olstad $^{8}$ & $89 \%$ & $9.80 \%$ \\
Forsyth-Fleck $^{9}$ & $43 \%$ & $4.20 \%$ \\
\hline
\end{tabular}

In Table 2, the experimental results had an accuracy of $85.2 \%$ and average processing time was $213.14 \mathrm{msec}$. per image.

\section{CONCLUSIONS AND FUTURE WORK}

This paper presented a new nude image classification technique where the main contribution was simplicity with accuracy that was still high within an acceptable processing time. The pixel properties of images such as those within an $\mathrm{YCbCr}$ range and eccentricity were examined. The images were converted from a RGB model into YCbCr model. Then each pixel was compared to an $\mathrm{YCbCr}$ range: $\mathrm{Y}>100,100<\mathrm{Cb}<120$ or $128<\mathrm{Cb}<130$ and $130<\mathrm{Cr}<160$.

According to Chai and Ngan paper ${ }^{1}, \%$ FACE_AREA range is between $110<\mathrm{Cb}<123$ and $136<\mathrm{Cr}<156$. \% AHB is therefore between $123<\mathrm{Cb}<$ 130 and $156<\mathrm{Cr}<160$. The experimental results showed that the accuracy was $85.2 \%$ and average processing time was $213.14 \mathrm{msec}$. per image.

From section 3.4 and 3.5, the decision rules were as follows;

1. If $(\% \mathrm{AHB}<0.4)$ and $(\operatorname{Rmax}>0.1)$ then Decision="Nude".

2. If $(\% \mathrm{AHB}<0.4)$ and $(\operatorname{Rmax}<=0.1)$ then Decision="Not Nude".

3. If $(\%$ AHB $>=0.4)$ and $(\%$ Face_Area $>0.3)$ then Decision="Not Nude"

4. If $(\% \mathrm{AHB}>=0.4)$ and $(\%$ Face_Area $<=0.3)$ then Decision="Nude"

In reality, there are some cases that an area of human skin in nude images is relatively low, such as with a body paint image or naked women under water. Moreover, a body paint image might be considered nude/not nude image in some cultures. Any picture that is not taken directly by a 
camera or is body painted would bring down the accuracy percentage. These kinds of images were considered as out of scope in this paper. In further study, a rough set or genetic algorithm are other ways to deal with vagueness.

\section{REFERENCES}

[1] D. Chai and King N. Ngan, Face segmentation using skin-color map in videophone applications. IEEE Transactions on circuits and systems for video technology, 9(4), 1999. http://dx.doi.org/10.1109/76.767122.

[2] M.Z. Talukder, A. Basak, and M. Shoyaib, Human skin detection. Global Journal of Computer Science and Technology Graphics and Vision, 13(3), Version 1.0, 2013

[3] J.-S. Leea, Y.-M. Kuob, P.-C. Chungb, and E-L. Chenc, Naked image detection based on adaptive and extensible skin color model. 40(8), 2261-2270, 2007. http://dx.doi.org/10.1016/j.patcog.2006.11.016.

[4] M.C. Shin, K.I. Chang, and L.V. Tsap, Does colorspace transformation make any difference on skin detection? Paper presented at the Sixth IEEE Workshop on Applications of Computer Vision, Orlando, FL, December 3-4, 2002. http://dx.doi.org/10.1109/ACV.2002.1182194.

[5] S. Karavarsamis, N. Ntarmos, K. Blekas, and I. Pitas, Detecting pornographic images by localizing skin ROIs, The International Journal of Digital Crime and Forensics. 5(1), 39-53, 2013. http://dx.doi.org/10.4018/jdcf.2013010103.

[6] Son Lam Phung, A. Bouzerdoum, and D. Chai, A novel skin color model in $\mathrm{YCbCr}$ color space and its application to human face detection. Paper presented at the International Conference on Image Processing, New York, USA, September 22-25, 2002. http://dx.doi.org/10.1109/ICIP.2002.1038016.

[7] B. Jedynak, H. Zheng, and M. Daoudi, Statistical models for skin detection. Paper presented at the IEEE Workshop on Statistical Analysis in Computer Vision, in Conjunction with CVPR, Madison, Wisconsin, USA, June 16-22, 2013. http://dx.doi.org/10.1109/CVPRW.2003.10094.

[8] W.A. Arentz and B. Olstad, Classifying offensive sites based on image content. Computer Vision and Image Understanding, 94(1-3), 295-310, 2004. http://dx.doi.org/10.1016/j.cviu.2003.10.007.

[9] M.M. Fleck and D.A. Forsyth, Automatic detection of human nudes. International Journal of Computer Vision, 32(1), 63-77. 1999. 